\title{
On Poisson Structure and Curvature
}

\author{
J. Madore \\ Laboratoire de Physique Théorique et Hautes Energies* \\ Université de Paris-Sud, Bât. 211, F-91405 Orsay \\ June, 1997
}

\begin{abstract}
We consider a curved space-time whose algebra of functions is the commutative limit of a noncommutative algebra and which has therefore an induced Poisson structure. In a simple example we determine a relation between this structure and the Riemann tensor.
\end{abstract}

LPTHE Orsay $97 / 25$

*Laboratoire associé au CNRS, URA D0063 


\section{Motivation and notation}

One of the more interesting problems of noncommutative geometry (in physics) is the study of the commutative ('classical') limit. In a previous article (Dubois-Violette et al. 1997) we considered the case where the commutative limit was Poincaré invariant and we analyzed in detail the Poisson structure which necessarily remains as a 'shadow'. Here we suppose that the limit is a curved manifold and we discuss the relation between the Poisson structure and the curvature.

Let $\mathcal{A}=\mathcal{C}(V)$ be the algebra of (smooth, complex-valued) functions on a (real) space-time $V$, which for simplicity we shall suppose diffeomorphic to $\mathbb{R}^{4}$, and let $\Omega^{*}(\mathcal{A})$ be the algebra of de Rham differential forms. The space $\Omega^{1}(\mathcal{A})$ of 1 -forms is free of rank 4 as an $\mathcal{A}$-module; there exists a globally defined moving frame $\theta^{\alpha}$. It can be argued that space-time should be more properly described by a noncommutative $*$-algebra $\mathcal{A}_{k}$ over the complex numbers with four hermitian generators $q^{\lambda}$. The parameter $\hbar$ is a fundamental area scale which is presumably of the order of the Planck area $G \hbar$. We refer, for example, to Madore (1997) for a synopsis of the arguments. Let $\mathcal{A}_{0}$ be the commutative limit of $\mathcal{A}_{k}$. We shall suppose that the algebra $\mathcal{A}_{k}$ is a deformation of $\mathcal{A}_{0}$ in the sense of Gerstenhaber (1964). The relation between $\mathcal{A}_{k}$ and the classical space-time $V$ is given (Dubois-Violette et al. 1997) by the inclusion relation $\mathcal{A} \subset \mathcal{A}_{0}$. We shall assume that we can identify $\mathcal{A}_{0}=\mathcal{C}\left(V_{0}\right)$ as the algebra of (smooth, complexvalued) functions on a (real) parallelizable manifold $V_{0}$ and that there is a projection of $V_{0}$ onto $V$.

One can consider $V_{0}$ as a Kaluza-Klein extension of $V$ by a manifold which is perhaps of infinite dimension and in general not compact. It should be stressed however that $V_{0}$ is a mathematical fiction. The 'real' world is described by the algebra $\mathcal{A}_{k}$; it is this algebra which we consider to be the correct Kaluza-Klein extension of $V$ (Madore 1989b, Madore \& Mourad 1996a). The difference in dimension between $V_{0}$ and $V$ is one of the measures of the extent to which the verb 'to quantize' as applied to the coordinates of $V$ is a misnomer; one could in extremis 'quantize' the coordinates of $V_{0}$. The observables will be some subset of the hermitian elements of $\mathcal{A}_{k}$. We shall not discuss this problem here; we shall implicitly suppose that all hermitian elements of $\mathcal{A}_{\hbar}$ are observables, including the 'coordinates'. We shall not however have occasion to use explicitly this fact. If there is a gravitational field then there must be some source, of characteristic mass $\mu$. If $\mu^{2} \hbar$ tends to zero with $\hbar$ then $V_{0}$ will be without curvature. We are interested here in the case in which $\mu^{2} \hbar$ tends to some finite non-vanishing value as $k \rightarrow 0$.

Quite generally the commutator of $\mathcal{A}_{\hbar}$ defines a Poisson structure (Vaisman 1994) on $V_{0}$. We have given arguments in the past (Madore \& Mourad 1996b), based on simple models, that a differential calculus over $\mathcal{A}_{\hbar}$ should determine a metric-compatible torsion-free linear connection on $V_{0}$. It is natural then that there should be a relation between the Poisson structure and the curvature of the connection. We can show that certain natural hypotheses on the differential calculus yield in fact relations between the two. We have been however unable so far to present a realistic gravitational field explicitly as the 'shadow' of a differential calculus over a noncommutative algebra.

In the next section we make some general remarks concerning the problem of 'quantization' of space-time. In Section 3 we discuss in particular the case of a dynamical space-time which is asymptotically flat. Greek indices take values from 0 to 3 ; the first half of the alphabet is used to index frames and the second half to index generators. Latin indices take values from 0 to $n-1$. The bracket $[\xi, \eta]$ of two elements $\xi$ and $\eta$ of a graded algebra is a graded bracket. 


\section{The general formalism}

We define 6 more elements $q^{\mu \nu}$ of $\mathcal{A}_{\hbar}$ by the relations

$$
\left[q^{\mu}, q^{\nu}\right]=i \hbar q^{\mu \nu}
$$

The details of the structure of $\mathcal{A}_{\hbar}$ will be contained, for example, in the commutation relations $\left[q^{\lambda}, q^{\mu \nu}\right]$. The $q^{\mu \nu}$ can be also considered as extra generators and the Equations (2.1) as extra relations. In this case the $q^{\mu \nu}$ cannot be chosen arbitrarily. They must satisfy the four Jacobi identities:

$$
\left[q^{\lambda}, q^{\mu \nu}\right]+\left[q^{\mu}, q^{\nu \lambda}\right]+\left[q^{\nu}, q^{\lambda \mu}\right]=0 .
$$

We define recursively an infinite sequence of elements by setting, for $p \geq 1$,

$$
\left[q^{\lambda}, q^{\mu_{1} \cdots \mu_{p}}\right]=i \hbar q^{\mu_{1} \cdots \mu_{(p+1)}} .
$$

Several structures have been considered in the past, by Snyder (1947), Madore (1989a, 1995) and Doplicher et al. (1994). With our choice of normalization $q^{\mu_{1} \cdots \mu_{p}}$ has units of mass to the power $p-2$.

We shall assume that for the description of a generic gravitational field the appropriate algebra $\mathcal{A}_{\hbar}$ has a trivial center $\mathcal{Z}_{\hbar}$ :

$$
\mathcal{Z}_{k}=\mathbb{C}
$$

The only argument we have in favour of this assumption is the fact that it could be argued that if $\mathcal{Z}_{k}$ is greater then $\mathbb{C}$ then the 'quantization' has been only partial. It implies of course that the sequence of $q^{\mu_{1} \cdots \mu_{p}}$ never ends, although all these elements need not be independent.

The simplest example of an algebra is the tensor algebra $\mathcal{A}_{u}$ over the vector space spanned by the 4 generators $q^{\mu}$. This algebra has a natural filtration $\mathcal{F}_{u}^{p}$ in powers of $\hbar$. One defines $\mathcal{F}_{u}^{0}$ as the symmetric algebra over the generators. We can identify this with the algebra of polynomials on the classical space-time $V$ and, by completion, with the algebra of smooth functions. One defines $\mathcal{F}_{u}^{1}$ as the kernel of the projection of $\mathcal{A}_{u}$ onto $\mathcal{F}_{u}^{0}$. It is an ideal of $\mathcal{A}_{u}$ each of whose elements contains at least one commutator. One defines $\mathcal{F}_{u}^{p}$ as the ideal of those elements which contain at least $p$ commutators. The most general algebra $\mathcal{A}_{k}$ is a quotient of $\mathcal{A}_{u}$ by some ideal $\mathcal{I}$ and the filtration of $\mathcal{A}_{u}$ defines a corresponding filtration $\mathcal{F}_{k}^{p}$ of $\mathcal{A}_{k}$. There is an intimate connection between the dimension of $V_{0}$ and the 'size' of $\mathcal{I}$. We set

$$
x^{\mu_{1} \cdots \mu_{p}}=\lim _{k \rightarrow 0} q^{\mu_{1} \cdots \mu_{p}} .
$$

A set of independent elements of the complete set of the $x^{\mu_{1} \cdots \mu_{p}}$ are local coordinates of $V_{0}$. If $V$ is Minkowski space-time then the condition of Lorentz invariance in the commutative limit forces $x^{\lambda}$ and at least 4 of the 6 coordinates $x^{\mu \nu}$ to be independent (Doplicher et al. 1994). In general the $x^{\mu_{1} \cdots \mu_{p}}$ for $p \geq 3$ can at least in part be functions of $x^{\lambda}$ and $x^{\mu \nu}$. This will depend on the structure of the ideal $\mathcal{I}$. If $\mathcal{I}=0$ then all the $x^{\mu_{1} \cdots \mu_{p}}$ for $p \geq 3$ are independent coordinates and $\operatorname{dim} V_{0}=\infty$. If on the other hand $x^{\mu_{1} \cdots \mu_{p}}=x^{\mu_{1} \cdots \mu_{p}}\left(x^{\lambda}\right)$ for all $p \geq 2$ then $V_{0}=V$.

Let $f$ and $g$ be elements of $\mathcal{A}_{k}$. To simplify the notation we use the same symbol to designate the corresponding limit functions on $V_{0}$. The Poisson bracket is given by

$$
\{f, g\}=\lim _{\hbar \rightarrow 0} \frac{1}{i \hbar}[f, g] .
$$


The restriction of the bracket to $\mathcal{A}$ is given by

$$
\{f, g\}=x^{\mu \nu} \partial_{\mu} f \partial_{\nu} g .
$$

Let $\Omega^{*}\left(\mathcal{A}_{k}\right)$ be a differential calculus over $\mathcal{A}_{k}$. We shall assume that $\Omega^{*}\left(\mathcal{A}_{k}\right)$ is non-degenerate in the sense that if $d f=0$ then $f \in \mathcal{Z}_{k}$. This is a natural assumption. It is easy to see that $\Omega^{1}\left(\mathcal{A}_{\hbar}\right)$ is generated by the $d q^{\lambda}$ as a bimodule. It is typical in fact of noncommutative differential calculi that $\Omega^{1}\left(\mathcal{A}_{k}\right)$ have one generator as a bimodule and that the $d q^{\lambda}$ can be written in turn as a commutator of a sort of generalized 'Dirac operator' (Connes 1986). We shall suppose that $\Omega^{1}\left(\mathcal{A}_{k}\right)$ is a free left (and right) $\mathcal{A}_{k^{-}}$ module of rank $n$ with a basis $\theta^{a}, 0 \leq a \leq n-1$, which commutes with the generators $q^{\lambda}$ of $\mathcal{A}_{k}$ :

$$
\left[q^{\lambda}, \theta^{a}\right]=0 .
$$

In view of the fact that we suppose that our algebras are 'quantizations' of parallelizable manifolds this is a natural assumption. We consider $\theta^{a}$ as the noncommutative analogue of a moving frame. We use the word 'frame' or 'Stehbein' since, as there are no points, there can be no 'movement'. It is easy to see that $n$ must be bounded below by the dimension of $V_{0}$ :

$$
n \geq \operatorname{dim} V_{0} \text {. }
$$

A rather trivial example of a differential calculus is the universal differential calculus which exists over every algebra. Its structure as a graded algebra is rather trivial and it is comforting to know that it admits naturally a trivial torsion-free linear connection (Madore \& Mourad 1996b). If we consider only differential calculi which are based on sets of derivations (See, for example, Dimakis \& Madore 1996) then the dual basis will automatically satisfy the condition (2.7). Of course any moving frame on $V_{0}$ satisfies an analogous condition. If $\theta^{\alpha}=d x^{\alpha}$ on an ordinary manifold then the associated torsionfree linear connection is flat. The noncommutative equivalent (2.7) would imply that $d q^{\mu_{1} \cdots \mu_{p}}=0$ for all $p \geq 2$. Because of our assumptions this means that $q^{\mu_{1} \cdots \mu_{p}}=0$ for all $p \geq 2$ and that the algebra is commutative.

An interesting question is the relation between the limit

$$
\Omega_{0}^{*}=\lim _{k \rightarrow 0} \Omega^{*}\left(\mathcal{A}_{k}\right)
$$

of a differential calculus over $\mathcal{A}_{\star}$ and the de Rham differential calculus $\Omega^{*}\left(V_{0}\right)$ over $V_{0}$. The universal calculus $\Omega_{u}^{*}\left(\mathcal{A}_{k}\right)$ obviously does not have $\Omega^{*}\left(V_{0}\right)$ as a limit. It would seem that $(2.7)$ is a necessary condition for this to be true if $V_{0}$ is parallelizable. It is not obvious that the dependence on a set of derivations is a sufficient condition. Suppose that $\Omega_{0}^{*}=\Omega^{*}\left(V_{0}\right)$. The commutator on $\Omega^{*}\left(\mathcal{A}_{k}\right)$ induces, in the commutative limit, a Poisson bracket on $\Omega^{*}\left(V_{0}\right)$. Let $\xi$ and $\eta$ be two elements of $\Omega^{*}\left(\mathcal{A}_{k}\right)$ which to be specific we shall suppose are 1-forms. We use the same symbol to designate the corresponding de Rham 1-forms. This notation can be misleading since it can happen (Madore 1995) that as an element of $\Omega^{1}\left(\mathcal{A}_{k}\right) \xi$ is exact but that as element of $\Omega^{1}\left(V_{0}\right)$ it is only closed. The Poisson bracket of $\xi$ and $\eta$ is given by the same formula (2.5) as for the functions. Obviously the Jacobi identity remains satisfied but the bracket of two 1 -forms need not be an element of $\Omega^{*}\left(V_{0}\right)$. To see this we write $\xi=\xi_{a} \theta^{a}$ and $\eta=\eta_{a} \theta^{a}$. Then

$$
[\xi, \eta]=\left[\xi_{a}, \eta_{b}\right] \theta^{a} \theta^{b}+\eta_{b} \xi_{a}\left[\theta^{a}, \theta^{b}\right] .
$$

The first term on the right-hand side tends obviously to the element $\left\{\xi_{a}, \eta_{b}\right\} \theta^{a} \theta^{b}$ of $\Omega^{2}\left(V_{0}\right)$. The limit of the second term however will be in general a more general element of $\Omega^{1}\left(V_{0}\right) \otimes \Omega^{1}\left(V_{0}\right)$. There is an interesting case in which a 'twisted' bracket $\{\xi, \eta\}_{C}$ 
can be defined (Chu et al. 1996) which takes its values in $\Omega^{*}\left(V_{0}\right)$. We suppose that the $\theta^{a}$ satisfy relations of the form (Dimakis \& Madore 1996)

$$
\theta^{a} \theta^{b}+C_{c d}^{a b} \theta^{c} \theta^{d}=0
$$

where the $C^{a b}{ }_{c d}$ are elements of the center of the algebra such that

$$
\lim _{k \rightarrow 0} C^{a b}{ }_{c d}=\delta_{c}^{b} \delta_{d}^{a}
$$

Then we can define

$$
\{\xi, \eta\}_{C}=\lim _{k \rightarrow 0} \frac{1}{i \hbar}\left(\xi_{a} \eta_{b}-C^{c d}{ }_{a b} \xi_{c} \eta_{d}\right) \theta^{a} \theta^{b}
$$

There always exist elements $\theta_{a}^{\mu_{1} \cdots \mu_{p}} \in \mathcal{A}_{\hbar}$ such that

$$
d q^{\mu_{1} \cdots \mu_{p}}=\theta_{a}^{\mu_{1} \cdots \mu_{p}} \theta^{a} .
$$

We have remarked that $\Omega^{1}\left(\mathcal{A}_{k}\right)$ is generated by the 4 elements $d q^{\lambda}$ as an $\mathcal{A}_{k}$-bimodule. We shall suppose that there exists a subset $d q^{i}$ of the $d q^{\mu_{1} \cdots \mu_{p}}$, with $0 \leq i \leq n-1$, which are also generators of $\Omega^{1}\left(\mathcal{A}_{k}\right)$ as a (left or right) $\mathcal{A}_{k^{-}}$-module. This means that there exist elements $\theta_{i}^{a}$ and $\theta_{i}^{\prime a}$ in $\mathcal{A}_{k}$ such that one can write

$$
\theta^{a}=\theta_{i}^{a} d q^{i}=d q^{i} \theta_{i}^{\prime a}
$$

It follows then that

$$
d q^{i} f=\theta_{a}^{i} \theta^{a} f=\theta_{a}^{i} f \theta^{a}=\left(\theta_{a}^{i} f \theta_{j}^{a}\right) d q^{j} .
$$

An element $f$ on the right of $d q^{i}$ can always be shifted to the left. An explicit counterexample of this is given in Section IV of the article by Dimakis \& Madore (1996).

As an example choose $n=10$ and set $\theta^{a}=\left(\theta^{\alpha}, \theta^{\beta \gamma}\right)$ with the second component antisymmetric in its two indices. We have then the expansion

$$
\theta^{\alpha}=\theta_{\lambda}^{\alpha} d q^{\lambda}+\frac{1}{2} \theta_{\mu \nu}^{\alpha} d q^{\mu \nu}, \quad \theta^{\beta \gamma}=\theta_{\lambda}^{\beta \gamma} d q^{\lambda}+\frac{1}{2} \theta_{\mu \nu}^{\beta \gamma} d q^{\mu \nu}
$$

We derive therefore from (2.7) the relations

$$
\theta_{\nu}^{\alpha}\left[q^{\mu}, d q^{\nu}\right]+\left[q^{\mu}, \theta_{\nu}^{\alpha}\right] d q^{\nu}+\frac{1}{2} \theta_{\rho \sigma}^{\alpha}\left[q^{\mu}, d q^{\rho \sigma}\right]+\frac{1}{2}\left[q^{\mu}, \theta_{\rho \sigma}^{\alpha}\right] d q^{\rho \sigma}=0
$$

and

$$
\theta_{\nu}^{\beta \gamma}\left[q^{\mu}, d q^{\nu}\right]+\left[q^{\mu}, \theta_{\nu}^{\beta \gamma}\right] d q^{\nu}+\frac{1}{2} \theta_{\rho \sigma}^{\beta \gamma}\left[q^{\mu}, d q^{\rho \sigma}\right]+\frac{1}{2}\left[q^{\mu}, \theta_{\rho \sigma}^{\beta \gamma}\right] d q^{\rho \sigma}=0
$$

These equations can in principle be solved to yield expressions for the commutators $\left[q^{\mu}, d q^{\nu}\right]$ and $\left[q^{\lambda}, d q^{\mu \nu}\right]$.

We have not succeeded in constructing a linear connection compatible with this frame in the sense of Madore \& Mourad (1996b). We have chosen $n=10$ so that there will be the correct number of degrees of freedom to yield a general metric but this is not of course a sufficient condition. It must be shown that the frame is dual to a set of derivations which satisfies compatibility conditions. We cannot show that the Riemann tensor we use below is is necessarily the limiting curvature of a linear connection on a noncommutative structure. 


\section{The asymptotically flat case}

Consider now a differential calculus $\Omega^{*}\left(\mathcal{A}_{k}\right)$ over an algebra $\mathcal{A}_{k}$ which is asymptotically flat in the sense that in the commutative limit there exists a radial coordinate $r$ such that

$$
\theta_{\lambda}^{\alpha}=\delta_{\lambda}^{\alpha}+o\left(r^{-1}\right), \quad \theta_{\mu \nu}^{\alpha}=o\left(r^{-1}\right) .
$$

Because of (2.8) the dimension of $V_{0}$ is at most equal to 10 . Let $\left(x^{\lambda}, x^{\mu \nu}\right)$ be local coordinates and $f$ an arbitrary element of $\mathcal{A}_{k}$. We can write then in the 'quasi-classical' approximation

$$
\left[q^{\lambda}, f\right]=i \hbar x^{\lambda \mu} \partial_{\mu} f+\frac{1}{2} i \hbar x^{\lambda \mu \nu} \partial_{\mu \nu} f+o\left(\hbar^{2}\right) .
$$

On the right-hand side one can consider $f$ as a function on $V_{0}$. We deduce from (2.12) in the limit $k \rightarrow 0$ that

$$
\begin{aligned}
d x^{\mu \nu}+x^{[\mu \rho} \partial_{\rho} \theta_{\sigma}^{\nu]} d x^{\sigma} & +\frac{1}{2} x^{[\mu \rho \sigma} \partial_{\rho \sigma} \theta_{\tau}^{\nu]} d x^{\tau}+\frac{1}{2} \theta_{\rho \sigma}^{\alpha}\left\{q^{\mu}, d q^{\rho \sigma}\right\} \\
& +\frac{1}{2} x^{[\mu \lambda} \partial_{\lambda} \theta_{\rho \sigma}^{\nu]} d x^{\rho \sigma}+\frac{1}{2} x^{[\mu \rho \sigma} \partial_{\rho \sigma} \theta_{\lambda \tau}^{\nu]} d x^{\lambda \tau}=0
\end{aligned}
$$

and we conclude that

$$
d x^{\mu \nu}=o\left(r^{-1}\right)
$$

Therefore the extra variables are dependent,

$$
x^{\mu \nu}=x^{\mu \nu}\left(x^{\lambda}\right)
$$

and the Poisson structure varies from point to point. We have $V_{0}=V$ and $\operatorname{dim} V_{0}=4$. Using (3.4) we can conclude that the last 4 terms in (3.3) are of order $o\left(r^{-2}\right)$ and we can rewrite $(3.3)$ as

$$
d x^{\mu \nu}+x^{[\mu \rho} \partial_{\rho} \theta_{\sigma}^{\nu]} d x^{\sigma}=o\left(r^{-2}\right) .
$$

This system of equations has integrability conditions which yield a relation between the symplectic structure and the curvature.

One can be more explicit when the gravitational field is dynamical with a smooth asymptotic expansion along a family of retarded null hypersurfaces. We can write then the derivatives of the components $\theta_{\mu}^{\alpha}$ of the moving frame on $V$ to leading order in the asymptotic expansion as

$$
\partial_{\lambda} \theta_{\mu}^{\alpha}=p_{\lambda} \dot{\theta}_{\mu}^{\alpha}+o\left(r^{-2}\right)
$$

with $p_{\lambda}$ a null vector and the dot derivation with respect to the function which parameterizes the family of hypersurfaces. Then Equation (3.6) becomes

$$
\dot{x}^{\mu \nu} p_{\sigma}+x^{[\mu \rho} p_{\rho} \dot{\theta}_{\sigma}^{\nu]}=o\left(r^{-2}\right) .
$$

Define $\tilde{p}^{\mu}=x^{\mu \nu} p_{\nu}$. Equation (3.7) has the particular solution

$$
\dot{x}^{\mu \nu}=o\left(r^{-2}\right), \quad \tilde{p}^{[\mu} \dot{\theta}_{\rho}^{\nu]}=o\left(r^{-2}\right)
$$

which yields the relation

$$
\tilde{p}_{[\mu} \ddot{g}_{\nu][\rho} \tilde{p}_{\sigma]}=o\left(r^{-2}\right) .
$$

On the other hand the asymptotic expression for the Riemann tensor is given by

$$
R_{\mu \nu \rho \sigma}=p_{[\mu} \ddot{g}_{\nu][\rho} p_{\sigma]}+o\left(r^{-2}\right) .
$$


To leading order the Riemann tensor is of null type with $p_{\mu}$ as principle vector. We see then from this simple example that there is an intimate relation between the Riemann tensor and the Poisson structure of space-time; we can write

$$
\left[q^{\mu}, q^{\nu}\right]=i \hbar x^{\mu \nu}+o\left(\hbar^{2}\right)
$$

where the right-hand side is strongly restricted if not determined by the Riemann tensor.

\section{Discussion}

We have found, to lowest order in the 'quantization' parameter an asymptotic form of the commutation relations which depends on the form of the asymptotic curvature. We are free to suppose that the commutation relations vanish at infinity but it would be quite consistent to suppose that they tend to constant values. An algebra has been proposed (Doplicher et al, 1994) for which the fundamental commutator of the generators is constant everywhere and yet possesses (Madore \& Mourad 1996b) a differential calculus with a torsion-free metric compatible flat linear connection. The most important assumption we have made concerning the differential calculus is contained in Equation (2.7).

\section{Acknowledgments}

Part of this research was done while the author was visiting the Ludwig-MaximiliansUniversität, München. He would like to thank J. Wess for his hospitality and interesting conversations.

\section{References}

Chu Chong-Sun, Ho Pei-Ming, Zumino B. 1996, Some Complex Quantum Manaifolds and their Geometry, Lectures given at the NATO Advanced Study Institute on Quantum Fields and Quantum Space Time, Cargese, hep-th/9608188.

Connes A. 1986, Non-Commutative Differential Geometry, Publications of the Inst. des Hautes Etudes Scientifique. 62257.

Dimakis A., Madore J. 1996, Differential Calculi and Linear Connections, J. Math. Phys. 374647.

Doplicher S., Fredenhagen K., Roberts, J.E. 1994, Spacetime quantization induced by classical gravity, Phys. Lett. B331 39.

Dubois-Violette M., Kerner R., Madore J. 1997, Shadow of Noncommutativity, Preprint LPTHE Orsay 96/06, q-alg/9702030.

Gerstenhaber M. 1964, On the deformation of rings and algebras, Ann. Math. 7959.

Madore J. 1989a, Non-Commutative Geometry and the Spinning Particle, XI Warsaw Symposium on Elementary Particle Physics, May, 1988, Kazimierz.

- 1989b, Kaluza-Klein Aspects of Noncommutative Geometry, Proceedings of the XVII International Conference on Differential Geometric Methods in Theoretical Physics, August, 1988, Chester. 
- 1995, An Introduction to Noncommutative Differential Geometry and its Physical Applications, Cambridge University Press.

- 1997, Fuzzy Space-time, Can. J. Phys. (to appear), gr-qc/9607065.

Madore J., Mourad. J. 1996a, Noncommutative Kaluza-Klein Theory Lecture given at the 5th Hellenic School and Workshops on Elementary Particle Physics, hepth/9601169.

Madore J., Mourad. J. 1996b, Quantum Space-Time and Classical Gravity, Preprint, LPTHE Orsay 95/56, gr-qc/9607060.

Snyder H.S. 1947, Quantized Space-Time, Phys. Rev. 7138.

Vaisman Izu 1994, Lectures on the Geometry of Poisson Manifolds, Birkhäuser Verlag, Basel. 